\title{
A REVIEW ON VARIOUS TYPES OF SOFTWARE DEFINED RADIOS (SDRS) IN RADIO COMMUNICATION
}

\author{
Vigneswara Rao Gannapathy ${ }^{1}$, Ahamed Fayeez Bin Tuani Ibrahim ${ }^{2}$, Zahriladha Bin Zakaria ${ }^{3}$, Abdul \\ Rani Bin Othman ${ }^{4}$, Nur Qalbi Binti Jalaudin ${ }^{5}$ \\ ${ }^{1}$ Lecturer, Faculty of Electronic \& Computer Engineering, Universiti Teknikal Malaysia Melaka (UTeM), \\ 76100 Melaka, Malaysia \\ ${ }^{2}$ Lecturer, Faculty of Electronic \& Computer Engineering, Universiti Teknikal Malaysia Melaka (UTeM), \\ 76100 Melaka, Malaysia \\ ${ }^{3}$ Lecturer, Faculty of Electronic \& Computer Engineering, Universiti Teknikal Malaysia Melaka (UTeM), \\ 76100 Melaka, Malaysia \\ ${ }^{4}$ Lecturer, Faculty of Electronic \& Computer Engineering, Universiti Teknikal Malaysia Melaka (UTeM), \\ 76100 Melaka, Malaysia \\ ${ }^{5}$ Student, Faculty of Electronic \& Computer Engineering, Universiti Teknikal Malaysia Melaka (UTeM), \\ 76100 Melaka, Malaysia
}

\begin{abstract}
Software Defined Radio (SDR) promises to deliver a cost effective and flexible solution by implementing a wide variety of wireless protocols in software. The SDR became more popular in recent years because of its abilities to realize many applications without a lot of efforts in the integration of different component. This software based radio device allows engineers to add more features to the communication system and implement any number of different signal processing elements or protocols without changing the original system hardware and its architecture. It provides a customizable and portable communications platform for many applications, including the prototyping and realization of wireless protocols and their performances. It is also able to interface with a separate hardware module to communicate over a real channel. In this article we described and compared the various SDRs that currently has been using by the researchers to study the performance of wireless protocol. Among the SDRs that we focused in this article are USRP, SORA, Air blue, SODA, and WARP.
\end{abstract}

Keywords: Software Defined Radio, USRP, SORA, Air blue, SODA, WARP - ****

\section{INTRODUCTION}

In 1992, Joseph Mitola proposed the basic concept of Software Defined Radio (SDR). SDR is the current radio communication which is implemented from the previous radio components, such as filters, modulation, demodulation, detectors, amplifiers, and mixers in software[1]. The motherboard is placed between the daughter board (RF front-end) and host computer at both transmitter and receiver. ADC/DAC changes the data format from analogue to digital and vice versa. Field Programmable Gate Array (FPGA) is used to execute high bandwidth mathematical calculations such as decimation, modulation/demodulation, and digital down conversion, digital up conversion and interpolation signal processing process. The wired is used to transmit and receive data between motherboard and host computer [2]. The architecture of SDR at the receiver and transmitter are shown in Figure 1.

The programmable hardware SDR has three approaches such as embedded digital signal processors (DSPs), Field Programmable Gate Arrays (FPGAs) and General-Purpose Processor (GPP)[3].

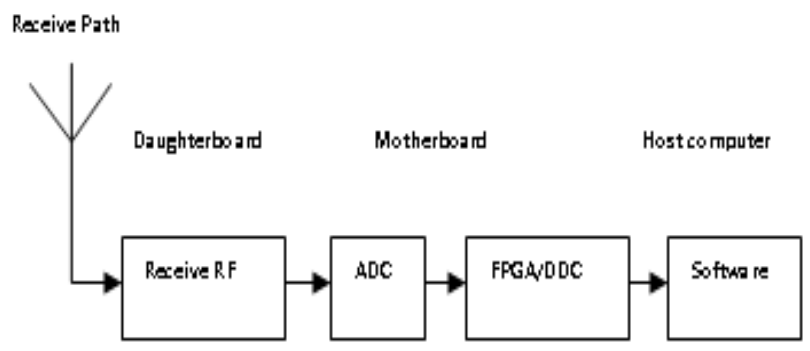

(a)

Trens mit Path

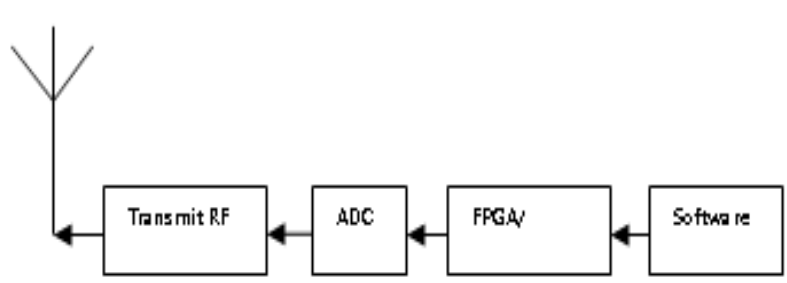

(b)

Fig -1: Basic SDR Architecture (a) Receiver (b) Transmitter 
The specialized processor in SDR is called DSP. The processing of these signals is needed to extract the information from the signal and it represents the signals digital as a sequence of numbers or symbols. The basic operations of the processor include some processes such as filtering, transformations, modulation, correlation and convolution[4].

FPGAs are semiconductor devices which consist of logic components and interconnect that are both programmable. The element can be combined to perform simple gate level logic operations such as AND, XOR, etc. The advantages of the architecture of an FPGA allow the designs to perform multiple computational operations in parallel. Parallelism enable substantial data throughput at relatively low lock rates [5].

The GPP are designed for computers which is personal computer /workstation. It is easier to program the platform and makes it flexible. However, high energy consumption is needed to achieve the performance of objective [6].

Currently SDR can be used to implement simple radio modem technologies such as Global System for Mobile Communications (GSM), Wide band Code Division Multiple Access (WCDMA), WiMAX, Wi-Fi and others.

This paper reviews on the comparison between various types of Software Defined Radio such as SDR USRP, SDR SORA, SDR AirBlue, SDR SODA and SDR WARP. The SDR Universal Software Radio Peripheral (USRP) will be explained in section II, while section III will discuss about SDR Software Radio (SORA). Next, SDR AirBlue will be discussed in section IV, and SDR Signal Processing OnDemand Architecture (SODA) will be described in section V. Lastly, SDR Wireless open-Access Research Platform (WARP) is discussed in section V1.

\section{SDR USRP}

The USRP is proposed by Ettus Research [7]. USRP is flexible platform and low cost. The hardware for USRP it consists of ADC/DAC, FPGA and USB controller [2].

USRP1 consist an Altera Cyclone FPGA. This version provides $128 \mathrm{MS} / \mathrm{s}$ dual DAC, $64 \mathrm{MS} / \mathrm{s}$ dual ADC and it provides data to host processers. USB 2.0 connectivity is used in this peripheral [8].

USRP2 consist of a Xilinx Spartan 3-2000 FPGA. This version provides $400 \mathrm{MS} / \mathrm{s}$ dual DAC, $100 \mathrm{MS} / \mathrm{s}$ dual ADC and to provide data to host processers using Gigabit Ethernet interface [9].

In USRP N Series, there are two types which are USRP N200 and USRP N210. USRP N200 consist a Xilinx Spartan 3A DSP. This version provides $400 \mathrm{MS} / \mathrm{s}$ dual DAC, $100 \mathrm{MS} / \mathrm{s}$ dual ADC and use Gigabit Ethernet interface offering data to host processors [10].
USRP N210 consist a Xilinx Spartan 3A DSP. This version provides $400 \mathrm{MS} / \mathrm{s}$ dual DAC, 100MS/s dual ADC and provides data to host processers by using Gigabit Ethernet interface[11]. USRP2 was no longer exist because it was not intended to replace the original USRP, which continue to be sold in parallel to the USRP2. It has been replaced USRP N200 and USRP N210. The comparison board resources between USRP1, USRP2, USRP N200 and USRP N210 are shown in Table 1 below:

Table 1: Comparison Board Resources between USRP1, USRP2, USRP N200 and USRP N210.

\begin{tabular}{|l|l|l|l|l|}
\hline & USRP1 & USRP 2 & $\begin{array}{l}\text { USRP } \\
\text { N200 }\end{array}$ & $\begin{array}{l}\text { USRP } \\
\text { N210 }\end{array}$ \\
\hline Interface & USB 2.0 & $\begin{array}{l}\text { Gigabit } \\
\text { Ethernet }\end{array}$ & $\begin{array}{l}\text { Gigabit } \\
\text { Ethernet }\end{array}$ & $\begin{array}{l}\text { Gigabit } \\
\text { Ethernet }\end{array}$ \\
\hline $\begin{array}{l}\text { Total Host } \\
\text { BW } \\
\text { (MSPS } \\
\text { 16b/8b) }\end{array}$ & $8 /^{*}$ & 16 & $50 / 100$ & $50 / 100$ \\
\hline $\begin{array}{l}\text { Daughter } \\
\text { card Slots }\end{array}$ & 2 & 2 & 1 & 1 \\
\hline $\begin{array}{l}\text { ADC } \\
\text { Resolution }\end{array}$ & 12 & 14 & 14 & 14 \\
\hline ADC Rate & 64 & 100 & 100 & 100 \\
\hline $\begin{array}{l}\text { DAC } \\
\text { Resolution }\end{array}$ & 14 & 16 & 16 & 16 \\
\hline DAC Rate & 128 & 400 & 400 & 400 \\
\hline $\begin{array}{l}\text { Internal } \\
\text { GPS } \\
\text { Disciplined } \\
\text { Oscillator }\end{array}$ & No & No & Yes & Yes \\
\hline $\begin{array}{l}\text { 1PPS/Ref } \\
\text { Inputs }\end{array}$ & No & Yes & Yes & Yes \\
\hline
\end{tabular}

\subsection{GNU Radio Toolkit}

GNU Radio is suggested by Eric Blossom with a group of developing research and design team from A.D.2000. GNU radio is an open source project for SDR platforms [1]. It can be used for Radio Frequency (RF) real time applications. The signal processing is written in $\mathrm{C}++$. The major application program offers the flow and connects the blocks via Phyton. This application is always chosen because of its ease of programming and flexibility [2].

\section{SDR SORA}

The SORA project started as a 'blue-sky' research project in the Wireless and Networking Group at Microsoft Research Asia (Beijing, China). The architectures of SDR SORA contain both software and hardware [13].

In the SORA architecture at the hardware components are a new radio control board ( $\mathrm{RCB}$ ) with an interchangeable radio front-end ( $\mathrm{RF}$ front-end). The contents of hardware element are Virtex-5 FPGA, PCIe-x8 interface and 256MB of DDR2 Synchronous Dynamic Random-Access Memory (SDRAM). The radio front-end is a hardware element which receives 
and/or transmits radio signals through an antenna. RF frontend is proposed by Rice University which is capable of transmitting and receiving a $20 \mathrm{MHzchannel}$ at $2.4 \mathrm{GHz}$ or $5 \mathrm{GHz}[14]$.

The SORA software works in $\mathrm{C}$, with some assembly for performance-critical processing. It offers necessary system services and programming support for implementing various wireless PHY and MAC protocols in a general-purpose operating systems[13].

\section{SDR AIRBLUE}

Air blue is developed by Nokia Inc. Airblue consists of a low-end Altera Cyclone III FPGA. The FPGA possess a direct connection to a $2.4 \mathrm{GHz}$. The baseband modulation is $40 \mathrm{MHz}$ and the RF front-end is $20 \mathrm{MHz}$. The host processor communicates using high-speed USB[15].

\section{SDR SODA}

SODA is proposed by ARM Ltd. It consists of four cores, each containing asymmetric dual pipelines which support scalar and 32 wide SIMD execution. The arithmetic units are customized for 16 bits while only a few ports are needed for the register files and software-controlled scratchpad memories [16].

SODA processing element (PE) has five main components which are: 1) a scalar pipeline for sequential operations. 2) a programmable DMA unit to transfer data between memories and interface with the outside system. 3) an address generation unit (AGU) pipeline for providing the addresses for local memory access. 4) two local scratch- pad for the SIMD pipeline and the scalar pipeline 5) a SIMD pipeline for supporting vector operations [17].

\section{SDR WARP}

This section will be discussed on SDR Wireless open-access Research Platform. It describes the architecture of SDR WARP and the key feature of SDR WARP includes FPGA board V1, FPGA board V2 and WARP V3. The key features of SDR WARP will be described briefly as in Table 2.
WARP is developed by RICE University research team [18] and distributed by Mango Communication. WARP is an extensible, scalable and programmable wireless tool [19]. It is able to transmit in the $2.4 \mathrm{GHz}$ to $2.5 \mathrm{GHz}$ and to 5.875 $\mathrm{GHz}$ range with up to a $40 \mathrm{MHz}$ bandwidth. The central in a WARP node is the FPGA board. Xilinx FPGA which is located in the heart of the board provides all the node's processing resources. It consists of a Xilinx FPGA and it provides daughter card slots that are compatible with all current WARP daughter card [20].

WARP FPGA board hardware version 1.2 consists of a Xilinx Virtex-II Pro FPGA. This version provides 10/100 Ethernet interface that is suitable for real-time communication between the wired network and wireless environment. There are four daughter card slots in a regular WARP [21] .

WARP FPGA board hardware version 2.2 consists of a Xilinx Virtex-4 FPGA. It offers 10/100/1000 Ethernet interface that is suitable for real-time communication between the wired network and wireless environment. It provides daughter card slots that are compatible with all current WARP daughter cards [22].

The latest generation of WARP hardware is WARP V3 which consists of a Xilinx Virtex-6 [23]. It integrates a high performance FPGA, two flexible RF interfaces and multiple peripherals to facilitate rapid prototyping of custom wireless design. The comparison board resources between FPGA board V1, FPGA board V2 and WARP V3 are shown in Table 2 below:

Table 2: Comparison Board Resources between FPGA board V1, FPGA board V2 and WARP V3.

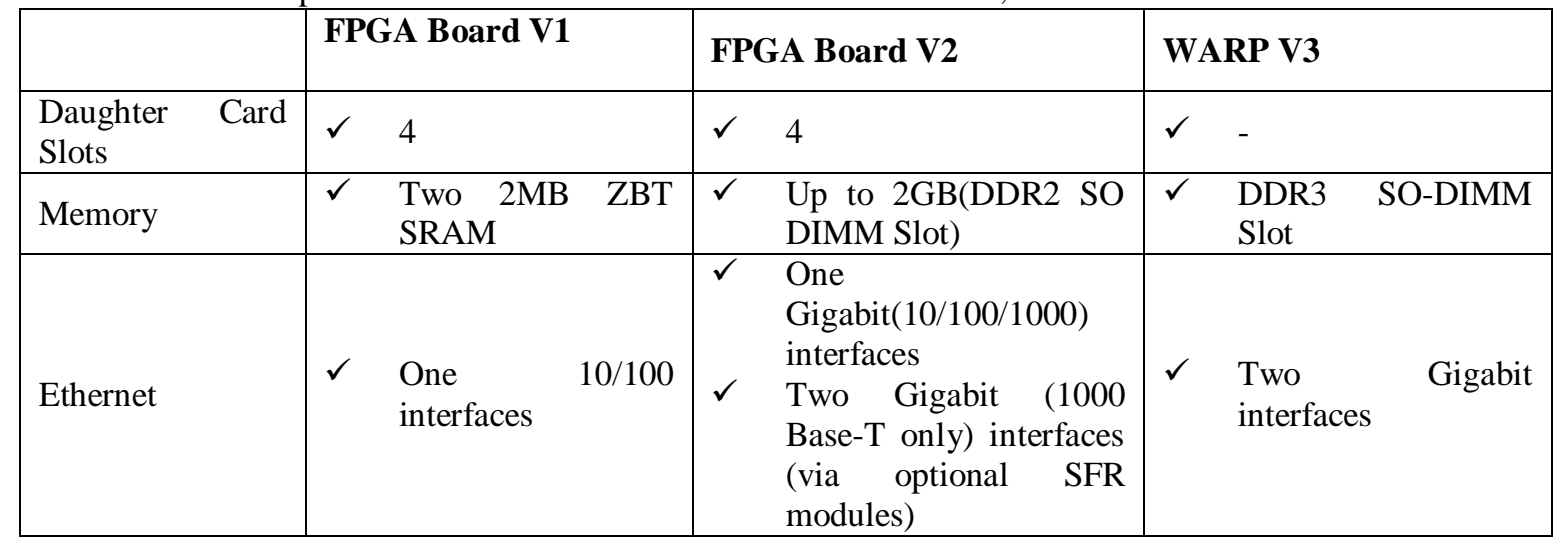




\begin{tabular}{|c|c|c|c|}
\hline $\begin{array}{l}\text { Multi Gigabit } \\
\text { Transceivers }\end{array}$ & $\begin{array}{ll}\checkmark \quad \text { 8HSSDC } 2 \text { ports (2 } \\
\text { populated) }\end{array}$ & $\begin{array}{ll}\checkmark & \text { 4HSSDC } 2 \text { Ports } \\
& \text { (Infiniband keyed) } \\
\checkmark & \text { 2 SATA Ports (1 Host, } 1 \\
& \text { Target) } \\
\checkmark & \text { 2 SFP ports }\end{array}$ & $\begin{array}{ll}\checkmark & 2.4 / 5 \mathrm{GHz} \\
& \text { transceiver(40MHz } \\
& \text { RF bandwidth) } \\
\checkmark & \text { 12-bit } 170 \quad \mathrm{MSps} \\
& \text { DAcs, 12-bit } \\
& \text { 100MSps ADCs }\end{array}$ \\
\hline MGT Clocking & $\begin{array}{ll}\checkmark & \text { One on board } \\
\text { oscillator }\end{array}$ & $\begin{array}{ll}\checkmark & \text { Flexible Design } \\
\checkmark & 3 \text { on-board oscillators } \\
\checkmark & \text { Daisy Chain support }\end{array}$ & $\begin{array}{ll}\checkmark & \text { Dual-band PA(20 } \\
& \text { dBm, Tx power) } \\
\checkmark & \text { Shared Clocking } \\
& \text { MIMO applications }\end{array}$ \\
\hline UART & $\begin{array}{l}\checkmark \quad \text { One RS232 (DB- } \\
\text { 9M Port) }\end{array}$ & $\begin{array}{lll}\checkmark & \text { One RS232 } & \text { (DB-9M } \\
& \text { Port) } \\
\checkmark & \text { One } \quad \text { USB/UART } \\
& \text { interfaces (FT 232R } \\
& \text { Transceiver) }\end{array}$ & $\begin{array}{ll}\text { FMC } & \text { HPC } \\
\text { expansion slot } & \end{array}$ \\
\hline User I/O & $\begin{array}{ll}\checkmark & 2 \text { Seven Segment } \\
& \text { Displays } \\
\checkmark & 4 \text { LEDs } \\
\checkmark & 16 \text { Bit TTL I/O } \\
\checkmark & 5 \text { Push Buttons }\end{array}$ & $\begin{array}{lll}\checkmark & 3 \text { Seven Segment } \\
& \text { Displays } \\
\checkmark & 16 \text { LEDs (6 Red/6 } \\
& \text { Green/4Yellow) } \\
\checkmark & 16 \text { Bit TTL I/O } \\
\checkmark & 5 \text { Push Buttons }\end{array}$ & $\begin{array}{ll}\checkmark & 2 \text { Seven Segment } \\
& \text { Displays } \\
\checkmark & \text { 12 LEDs } \\
\checkmark & \text { 4 Push buttons } \\
\checkmark & 4 \text { bit DIP switch } \\
\checkmark & \text { USB-UART } \\
\checkmark & 16-\text { bit 2.5v I/O } \\
& \text { header }\end{array}$ \\
\hline
\end{tabular}

The comparison board resources between Air Blue, SODA and WARP are shown in Table 3 below:

Table 3: Comparison Air Blue, SODA and WARP

\begin{tabular}{|c|c|c|}
\hline Air Blue & SODA & WARP \\
\hline 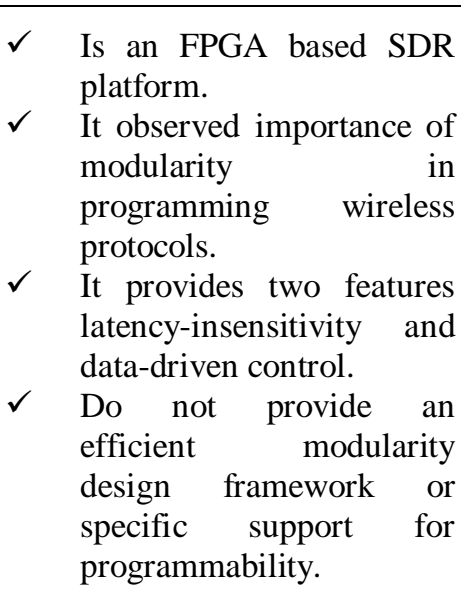 & $\begin{array}{l}\checkmark \text { The SIMD processor also } \\
\text { been employed for SDR } \\
\text { design to leverage the } \\
\text { advantage of high- } \\
\text { throughput processing. } \\
\checkmark \quad \text { it provides } \\
\text { programmability the } \\
\text { massive data parallel } \\
\text { computing model is not } \\
\text { suitable for wireless PHY } \\
\text { modules. } \\
\text { The GPU based } \\
\text { platforms are too power- } \\
\text { hungry for SDR system. }\end{array}$ & $\begin{array}{l}\checkmark \text { It can communicate } \\
\text { with a host } \\
\text { computer through } \\
\text { Ethernet } \\
\text { connections. } \\
\checkmark \quad \text { The latest version } \\
\text { of WARP equips a } \\
\text { relatively powerful } \\
\text { FPGA for signal } \\
\text { processing. } \\
\text { It also provide a } \\
\text { higher processing } \\
\text { capacity than } \\
\text { software based } \\
\text { SDR platforms. }\end{array}$ \\
\hline
\end{tabular}

The comparison board resources between USRP1, USRP N200, USRP N210 and SORA are shown in Table 4.

\section{CONCLUSION}

This article focused on the comparison of various types Software Defined Radios. Each of the architecture of SDR is discussed. It compares the performances of SDR in a wireless network. Based on the discussion of this article, SDR WARP V3 is the best choice because it integrates a high performance FPGA, two flexible RF interfaces and multiple peripherals facilitate rapid prototyping of custom wireless design. The advantage of SDR WARP, it is able to achieve the high symbol rates while execute all the signal processing inside the FPGA. The GNU Radio software can configure the USRP easily and ease to do programming. The drawback of GNU Radio is that it does not support 802.11 because of slow data transfer among the USB port to a personal computer. 
Table 4: Comparison USRP1, USRP N200, USRP N210 and SORA.

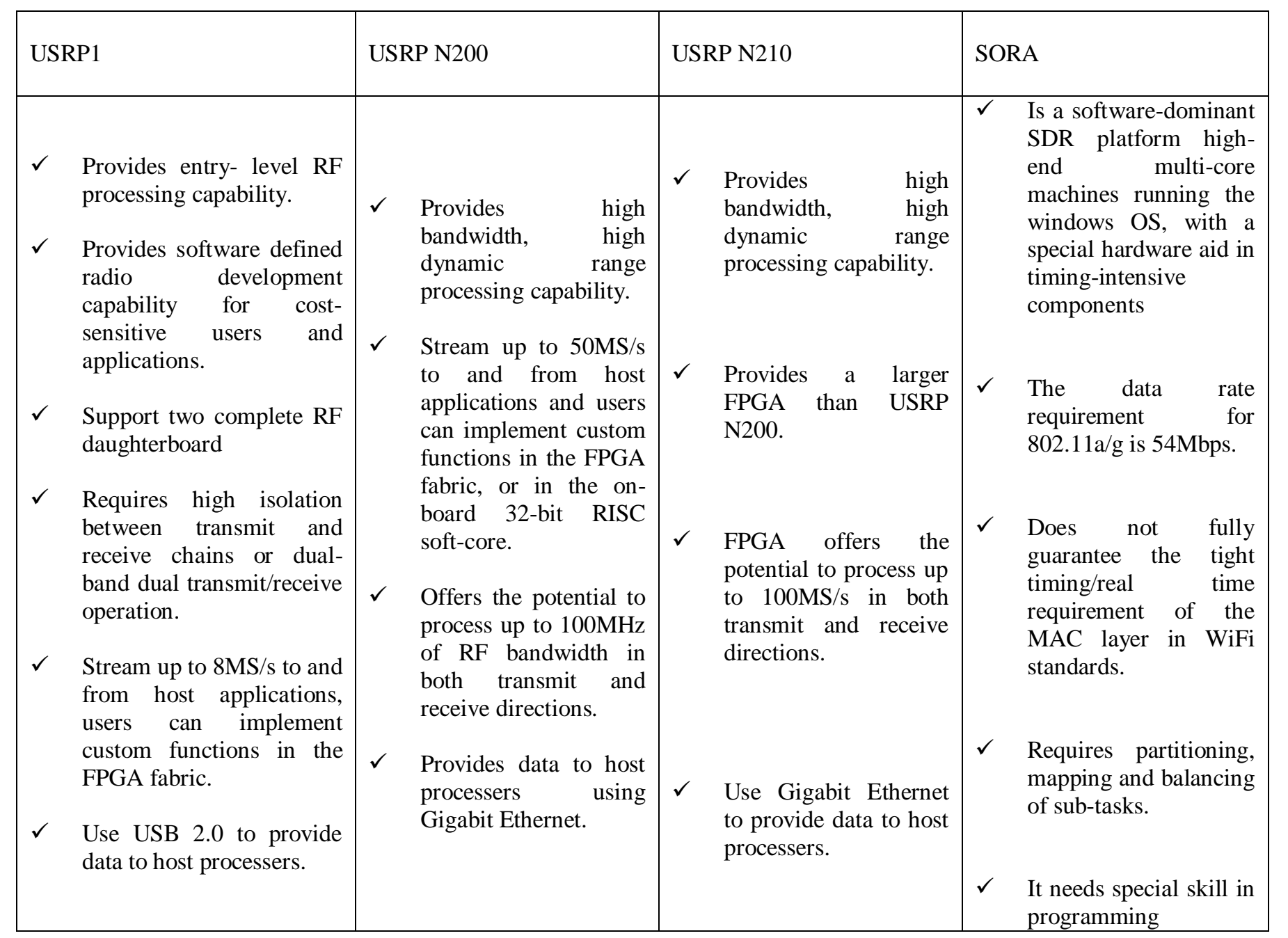

\section{ACKNOWLEDGEMENTS}

The authors would like to take this opportunity to thanks those who are contributes directly or indirectly in completion of this article and also for their constructive comments. In addition, the authors also would like to express our gratitude to Faculty of Electronic \& Computer Engineering, Universiti Teknikal Malaysia Melaka (UTeM), Melaka, Malaysia for the support and encouragement. The work in this paper was funded by Research Grant Scheme that offered to UTeM by Ministry of Higher Education, Malaysia.

\section{REFERENCES}

[1] C. Chen, F. Tseng, K. Chang, H. Chao, and J. Chen, "Reconfigurable Software Defined Radio and Its Applications," Tamkang J. Sci. Eng., vol. 13, no. 1, pp. 29-38, 2010.

[2] Z. Tong, M. S. Arifianto, and C. F. Liau, "Wireless Transmission using Universal Software Radio Peripheral," Int. Conf. Sp. Sci. Commun. 2009, pp. 1923, 2009.
[3] N. B. Truong and C. Yu, "Investigating Latency in GNU Software Radio with USRP Embedded Series SDR Platform," 2013 Eighth Int. Conf. Broadband Wirel. Comput. Commun. Appl., pp. 9-14, Oct. 2013.

[4] "Application Of Field Programmable Gate Array To DigitaL," J. Res. Natl. Dev., vol. 1, no. 9, pp. 37-46, 2011.

[5] A. Durke and H. Loomis, "NAVAL POSTGRADUATE," no. 03, p. 11, 2009.

[6] M. Dardaillon, K. Marquet, T. Risset, and A. Scherrer, "Software defined radio architecture survey for cognitive testbeds," 2012 8th Int. Wirel. Commun. Mob. Comput. Conf., pp. 189-194, Aug. 2012.

[7] A. A. Tabassam, F. A. Ali, S. Kalsait, and M. U. Suleman, "Building Software-Defined Radios in MATLAB Simulink - A Step Towards Cognitive Radios," 2011 UkSim 13th Int. Conf. Comput. Model. Simul., pp. 492-497, Mar. 2011. 
[8] J. R. Gutierrez-agullo, B. Coll-perales, and J. Gozalvez, “An IEEE 802 . 11 MAC Software Defined Radio Implementation for Experimental Wireless Communications and Networking Research," 2010 IFIP Wirel. Days, pp. 1-5, 2010.

[9] G. Berardinelli, P. Zetterberg, O. Tonelli, A. F. Cattoni, T. B. Sorensen, and P. Mogensen, "An SDR architecture for OFDM transmission over USRP2 boards," 2011 Conf. Rec. Forty Fifth Asilomar Conf. Signals, Syst. Comput., no. 1, pp. 965-969, Nov. 2011.

[10] J. R. Humphries and D. C. Malocha, "Software defined radio for passive sensor interrogation," $2013 \mathrm{Jt}$. Eur. Freq. Time Forum Int. Freq. Control Symp., pp. 270273, Jul. 2013.

[11] I. Galal, M. E. A. Ibrahim, and H. E. Ahmed, "Exploring Frequency Tuning Policies for USRP-N210 SDR Platform and GNU Radio," 2013 Conf. Des. Archit. Signal Image Process., 2013.

[12] H. Chang, H. Jin, X. Zhang, D. Liang, M. Peng, and W. Wang, "A Software Defined Radio Scheme for the Hierarchical Modulation Based Multimedia Broadcast System Using USRP," 2010 Int. Conf. Comput. Intell. Softw. Eng., pp. 1-4, Sep. 2010.

[13] J. Zhang, K. Tan, S. Xiang, Q. Yin, Q. Luo, Y. He, J. Fang, and Y. Zhang, "Experimenting software radio with the Sora platform," Proc. ACM SIGCOMM 2010 Conf. SIGCOMM - SIGCOMM '10, vol. 40, no. 4, pp. 469-470, 2010.

[14] K. Tan, J. Zhang, J. Fang, H. Liu, Y. Ye, S. Wang, Y. Zhang, H. Wu, W. Wang, and G. M. Voelker, "Sora : High Performance Software Radio Using General Purpose Multi-core Processors," Commun. ACM, pp. 99-107.

[15] M. C. Ng and K. Fleming, "AirBlue: A HighThroughput and Low-Latency Radio Prototyping Platform," 2011.

[16] Y. Lin, H. Lee, M. Woh, Y. Harel, S. Mahlke, and T. Mudge, "SODA: A Low-power Architecture For Software Radio," 33rd Int. Symp. Comput. Archit., pp. 89-101, 2006.

[17] Y. Lin and S. Mahlke, "SODA: A H IGH -P ERFORMANCE DSP A RCHITECTURE FOR S OFTWARE -D EFINED R ADIO," IEEE Micro, vol. 27, no. 1, pp. 114-123, 2007.

[18] A. Khattab, J. Camp, C. Hunter, P. Murphy, A. Sabharwal, and E. W. Knightly, "Demonstration Abstract: WARP - A Flexible Platform for CleanSlate Wireless Medium Access Protocol Design," Proceeding of Mobicom,Montreal Canada, pp. 4-6, 2007.
[19] K. Amiri, Y. Sun, P. Murphy, C. Hunter, J. R. Cavallaro, and A. Sabharwal, "WARP, a Modular Testbed for Configurable Wireless Network Research at Rice," IEEE Int. Conf. Microelectron. Syst. Educ. 07, pp. 53-54, 2007.

[20] P. Murphy, A. Sabharwal, and B. Aazhang, "DESIGN OF WARP: A WIRELESS OPEN-ACCESS RESEARCH PLATFORM," Eur. Signal Process. Conf., 2006.

[21] K.-H. Lee, A. Mate, and I.-T. Lu, "Practical implementation of time covariance based spectrum sensing methods using warp," 2011 IEEE Long Isl. Syst. Appl. Technol. Conf., pp. 1-5, May 2011.

[22] D. K. Artis, "Digital Modulations Using the Universal Software Radio Peripheral By," 2011.

[23] K. C. Garikipati, "Improving Transport Design for WARP SDR Deployments Categories and Subject Descriptors," Proc. ACM SIGCOMM Softw. Radio Implement. Forum (SRIF),2014, 2014.

[24] A. Sharma, V. Gelara, S. R. Singh, T. Korakis, and S. Panwar, "Implementation of a cooperative MAC protocol using a software defined radio platform," 2008 16th IEEE Work. Local Metrop. Area Networks, pp. 96-101, Sep. 2008.

[25] T. Korakis, M. Knox, E. Erkip, and S. Panwar, "Cooperative Network Implementation Using OpenSource Platforms," IEEE Commun. Mag., vol. 47, no. 2, pp. 134-141, 2009.

[26] V. R. Gannapathy, M. Kadim, B. Haji, M. Syahrir, B. Johal, L. K. Chuan, N. Bin Ramlit, and H. Mohamadt, "A Smooth Forwarding Operation in Wireless Mesh Network," IEEE 10th Malaysia International Conference on Communications (MICC), pp.83-87, October 2011.

[27] V. R. Gannapathy, M. R. Ahmad, M. K. Suaidi, M. S. Johal, and E. Dutkiewicz, "Concurrent MAC with short signaling for multi-hop wireless mesh networks," in 2009 International Conference on Ultra Modern Telecommunications and Workshops, 2009.

[28] V. R. Gannapathy, A. Fayeez, B. Tuani, Z. Bin Zakaria, A. Rani, B. Othman, and A. A. Latiff, "An Enhancement Of RTS / CTS Control Handshake In CSMA / CA Based MAC Protocol For An Efficient Packet Delivery Over Multihop Wireless Mesh Network ( WMN )," Int. J. Res. Eng. Technol. IJRET, vol. 02, no. 10, pp. 604-608, 2013.

[29] V. R. Gannapathy, M. R. Ahmad, M. K. Suaidi, M. S. Johal, "Performance Analysis of Concurrent Transmission with Reducing Handshakes in Multi-hop Wireless Mesh Networks (WMNs)", Journal of Telecommunication, Electronics and Computer 
Engineering (JTEC), ISSN: 2180 - 1843 Vol. 1 No. 1, pp. 69-76, July - December 2009.

\section{BIOGRAPHIES}

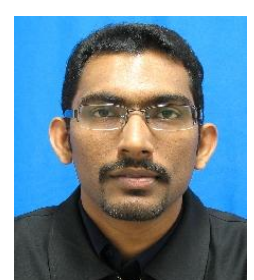

Engr. Vigneswara Rao Gannapathy currently serving as a lecturer in Universiti Teknikal Malaysia Melaka (UTeM) and he actively involves in research activities which is related to electronics and wireless networking. His research direction has focused on Wireless Mesh Networks which emerged as a key technology for next-generation wireless networking

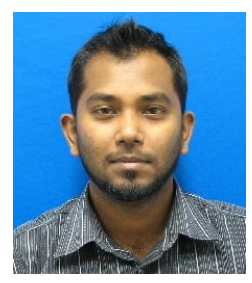

Ahamed Fayeez B Tuani Ibrahim, a Masters holder in Electronics \& Telecommunication, is a vivid computer networking enthusiast. His specific research interest includes low power network design and programming. He is a certified Cisco Network Analyst.

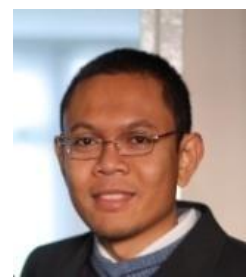

Dr. Zahriladha Zakaria, PhD, MIEEE, BEM, Grad IEM is currently working as a lecturer at the Faculty of Electronic and Computer Engineering, University Teknikal Malaysia Melaka. (UTeM). His research interests include a variety of microwave device development such as planar and non-planar microwave filters, amplifiers and antennas.

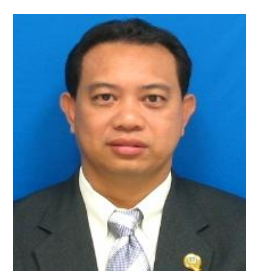

Associate Professor Dr. Abdul Rani Othman, $\mathrm{PhD}$, is currently working as lecturer at the Faculty of Electronic and Computer Engineering, University Teknikal Malaysia Melaka. (UTeM). His research interests include a variety of microwave device development, radio wave propagation, amplifiers and antennas. 